\title{
Dengue and yellow fever virus vectors: seasonal abundance, diversity and resting preferences in three Kenyan cities
}

\author{
Sheila B. Agha ${ }^{1,2^{*}}$ (D), David P. Tchouassi ${ }^{1}$, Armanda D. S. Bastos ${ }^{2}$ and Rosemary Sang ${ }^{1,3}$
}

\begin{abstract}
Background: The transmission patterns of dengue (DENV) and yellow fever (YFV) viruses, especially in urban settings, are influenced by Aedes (Stegomyia) mosquito abundance and behavior. Despite recurrent dengue outbreaks on the Kenyan coast, these parameters remain poorly defined in this and other areas of contrasting dengue endemicity in Kenya. In assessing the transmission risk of DENV/YFV in three Kenyan cities, we determined adult abundance and resting habits of potential Aedes (Stegomyia) vectors in Kilifi (dengue-outbreak prone), and Nairobi and Kisumu (no dengue outbreaks reported). In addition, mosquito diversity, an important consideration for changing mosquito-borne disease dynamics, was compared.

Methods: Between October 2014 and June 2016, host-seeking adult mosquitoes were sampled using $\mathrm{CO}_{2}$-baited BG-Sentinel traps (12 traps daily) placed in vegetation around homesteads, across study sites in the three major cities. Also, indoor and outdoor resting mosquitoes were sampled using Prokopack aspirators. Three samplings, each of five consecutive days, were conducted during the long-rains, short-rains and dry season for each city. Intercity and seasonal variation in mosquito abundance and diversity was evaluated using general linear models while mosquito-resting preference (indoors vs outdoors) was compared using Chi-square test.
\end{abstract}

Results: Aedes aegypti, which comprised 60\% $(n=7772)$ of the total 12,937 host-seeking mosquitoes collected, had comparable numbers in Kisumu $(45.2 \%, n=3513)$ and Kilifi $(37.7 \%, n=2932)$, both being significantly higher than Nairobi $(17.1 \%, n=1327)$. Aedes aegypti abundance was significantly lower in the short-rains and dry season relative to the long-rains $(P<0.0001)$. Aedes bromeliae, which occurred in low numbers, did not differ significantly between seasons or cities. Mosquito diversity was highest during the long-rains and in Nairobi. Only 10\% $(n=43)$ of the 450 houses aspirated were found positive for resting Ae. aegypti, with overall low captures in all areas. Aedes aegypti densities were comparable indoors/outdoors in Kilifi; but with higher densities outdoors than indoors in Kisumu and Nairobi.

Conclusions: The presence and abundance of Ae. aegypti near human habitations and dwellings, especially in Kilifi/ Kisumu, is suggestive of increased DENV transmission risk due to higher prospects of human vector contact. Despite low abundance of Ae. bromeliae suggestive of low YFV transmission risk, its proximity to human habitation as well as the observed diversity of potential YFV vectors should be of public health concern and monitored closely for targeted control. The largely outdoor resting behavior for Ae. aegypti provides insights for targeted adult vector control especially during emergency outbreak situations.

Keywords: Aedes aegypti, Aedes bromeliae, Vector abundance, Mosquito diversity, Resting preference, Urbanization, Kenya, Dengue and yellow fever risk

\footnotetext{
*Correspondence: sagha@icipe.org; aghasheila@yahoo.com

'International Centre of Insect Physiology and Ecology, P. O Box

30772-00100, Nairobi, Kenya

${ }^{2}$ Department of Zoology and Entomology, University of Pretoria, Private Bag

20, Hatfield 0083, South Africa

Full list of author information is available at the end of the article
} 


\section{Background}

Global epidemics of dengue and yellow fever are on the rise in most tropical and subtropical regions, with geographical expansion and increasing frequency of outbreaks being reported, especially in Africa [1-4]. Dengue virus (DENV) is the most rapidly spreading arbovirus in the world, with over 390 million global infections reported yearly $[5,6]$, whereas yellow fever virus (YFV) has a mortality rate of $20-50 \%$, rivaling that of Ebola virus. Both are arboviral diseases of major public health concern [4].

Since the first dengue outbreak in Kenya in 1982, which occurred in Kilifi and Malindi, subsequent outbreaks have mostly been limited to the Kenyan coast, especially in the urban city of Mombasa [7-9] and recently also affecting the Kenya-Somali border area [10]. This expansion in the geographical range of dengue outbreaks is of concern, as it highlights the potential for further spread. Urban yellow fever outbreaks are on the rise, as recently reported in Angola (Luanda) and Democratic Republic of Congo (Kinshasa), with cases imported into China and Kenya [4, 11, 12]. Although the last yellow fever outbreak in Kenya occurred in 19921993 [13], the disease is still considered a public health threat. This is driven in part by the potential for spread through national/international travel $[4,14]$ as well as the widespread presence of domestic/peri-domestic vectors, Aedes aegypti and Aedes bromeliae [15].

Yellow fever has continued to re-emerge in the last decade despite the availability of a safe and efficacious vaccine. Although a new dengue vaccine for use in emergency situations in highly endemic countries is currently available [16], the vaccine has not been licensed for use in many endemic countries, including Kenya. Current efforts for controlling dengue in Kenya therefore rely on reducing human-vector contacts as well as continuous suppression of the vector Ae. aegypti by targeting the immature stages. Based on previous studies carried out in Kenya, the most productive containers types for Aedes immatures were determined for targeted vector control, and the associated Stegomyia risk indices were established for assessing risk of DENV/YFV transmission $[9,17]$. However, studies focusing on adult mosquito populations are known to be more informative in estimating risk of transmission of these diseases [18]. Also, emergency interventions targeting adults remain crucial during outbreaks, the effectiveness of which depends on a good understanding of the adult abundance and resting behavior.

Aedes aegypti aegypti and Ae. aegypti formosus (hereafter referred to as $A$ e. aegypti) are genetically diverse forms of $A e$. aegypti, with the former being highly domesticated and often found in close association with humans, especially in urban settings, as opposed to the more zoophilic genetic form Ae. aegypti formosus [19]. As reported in large areas of Asia and South America, vectorial capacity of Ae. aegypti is influenced to a large extent not only by its extremely high human feeding tendency, but importantly, abundance and indoor resting habits $[1,20]$, which serve to enhance human-vector contact and maximize disease transmission. Surprisingly, knowledge of these attributes remains poorly defined in major dengue foci of Africa, especially in Kenya.

As part of an epidemiological assessment of risk of dengue and yellow fever outbreaks in Kenya, this study focused on estimating the abundance and diversity of potential DENV/YFV vectors in dengue-endemic (Kilifi County) and dengue-free (Kisumu and Nairobi County) cities in Kenya. We also assessed the domestic and peridomestic resting habits of potential DENV/YFV vectors in these cities, since the bionomics of a vector is known to shape the epidemiology of the disease. Data on the adult abundance and diversity can guide on the level of risk of transmission within each city, while identification of resting habits can be useful in vector control programs, providing baseline information on the different adult mosquito control strategies that can be implemented in case of an epidemic.

\section{Methods \\ Study site}

The study was carried out in three of the largest cities in Kenya, which despite all being major trade and travel hubs, differ with respect to dengue status with Kilifi being endemic to dengue, whereas there are no reports of dengue from Kisumu and Nairobi (Fig. 1). The capital Nairobi $\left(01^{\circ} 17^{\prime} \mathrm{S}, 36^{\circ} 48^{\prime} \mathrm{E}\right)$ is the largest city of Kenya and is located at an altitude of $1661 \mathrm{~m}$ above sea level (masl). Average monthly temperature ranges between 22 and $28{ }^{\circ} \mathrm{C}$. Kilifi County, situated at an altitude of 50 masl, occurs within the Coastal Region, incorporating Mombasa $\left(4^{\circ} 03^{\prime} \mathrm{S}, 39^{\circ} 40^{\prime} \mathrm{E}\right)$, the second largest city in Kenya. With an average monthly temperature range between 27 and $31{ }^{\circ} \mathrm{C}$, the Coastal Region has been endemic for dengue since 1982, and has experienced recent, as well as recurrent outbreaks in the last decade. Kisumu $\left(00^{\circ} 03^{\prime} \mathrm{S}, 34^{\circ} 45^{\prime} \mathrm{E}\right)$, the third largest city in Kenya is second only to Kampala in importance, within the Lake Victoria Region. It is situated at an altitude of 1131 masl and has an average monthly temperature range between 28 and $30{ }^{\circ} \mathrm{C}$.

Traps were set in four sub-locations within Kilifi County in Rabai including Bengo, Changombe, Kibarani, and Mbarakani. Similarly, trapping in Kisumu (Kisumu County), covered three sub-locations- Kajulu, Kanyakwar, and Nyalenda B. In Nairobi (Nairobi County), all traps were set in Githogoro. Sampling at sub-locations, conducted to ensure the widest possible coverage within 


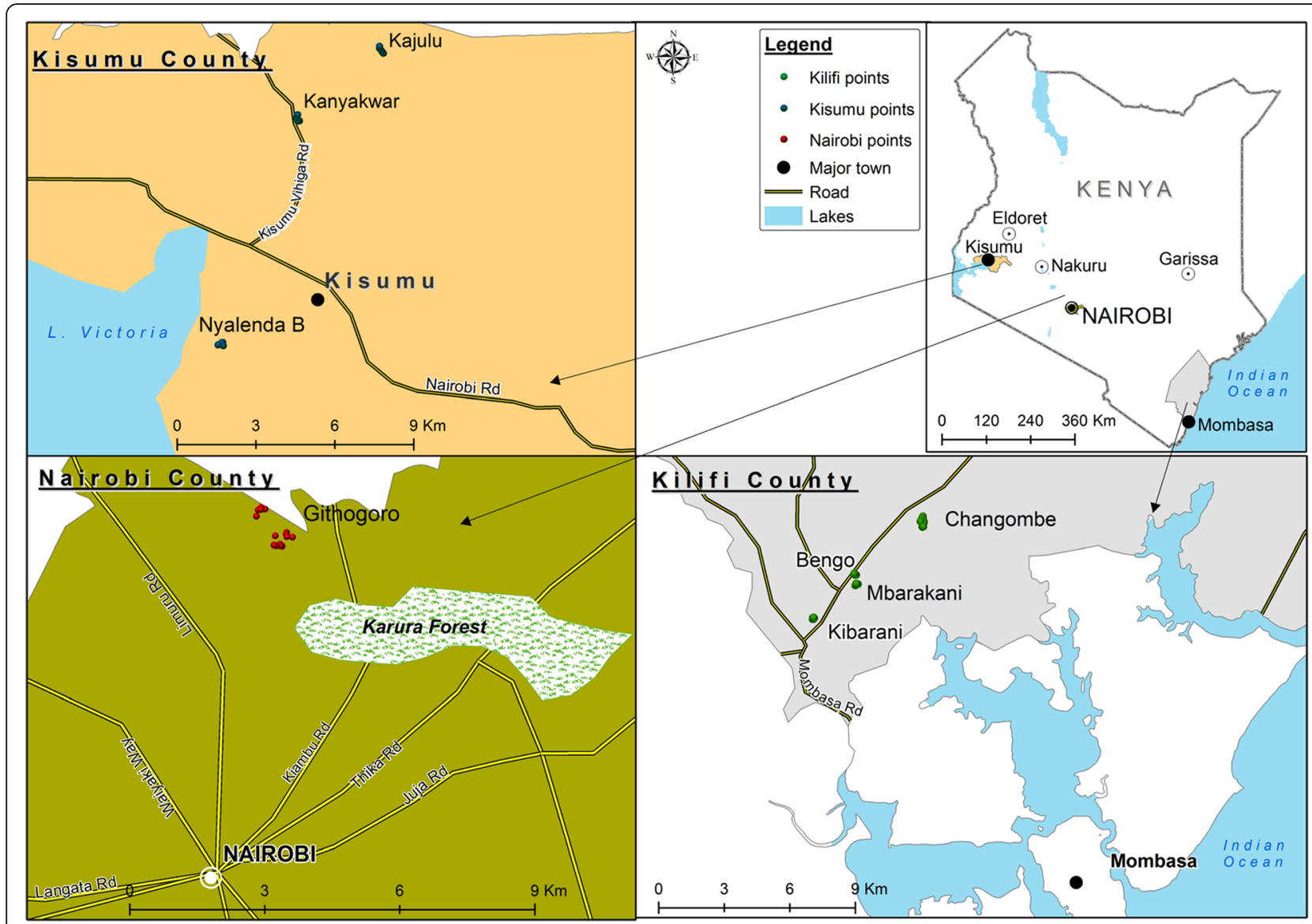

Fig. 1 Map showing the location of the study sites within Kilifi, Kisumu and Nairobi Counties of Kenya

each city, was balanced against logistical considerations, such as ease of accessibility to homesteads, particularly within Nairobi.

\section{Study design}

Sampling was conducted in the long-rains (April-June), short-rains (October-December) and dry season (JanuaryMarch or July-September), during 2014-2016. The seasons were primarily defined by the amount of rainfall. We obtained average rainfall data two weeks prior to mosquito sampling from the Kenya Meteorological Department, which during the long-rains was 12.4, 10.8 and $8.3 \mathrm{~mm}$; short-rains $5.5,4.0$ and $7.3 \mathrm{~mm}$ and the dry season $0,0.3$ and $0 \mathrm{~mm}$ in Kilifi, Kisumu and Nairobi, respectively.

Adult host-seeking mosquitoes were collected using BG-Sentinel traps (BioQuip Products, Rancho Dominguez, CA, USA), baited with $\mathrm{CO}_{2}$ supplied in the form of dry ice and placed outdoors in the vegetation around human habitations. The $\mathrm{CO}_{2}$ was dispensed by placing $\sim 0.5 \mathrm{~kg}$ in a thermos Igloo (2 l) per trap and suspended slightly above the trap entry. Traps (12 daily) were set up at $7 \mathrm{am}$ in the morning and retrieved at $6 \mathrm{pm}$ in the evening on the same day, for 5 consecutive days, in each season in each city, translating to a total of 540 BGSentinel traps being set (180 per city and 60 per season).

Collection of resting adult mosquitoes was performed using Prokopack aspirators (BioQuip Products, Rancho Dominguez, CA, USA) targeting Aedes resting mosquitoes indoors (sitting room, bedroom and kitchen) and outdoors (on nearby vegetation and the walls outside the house). Houses in each city were purposively selected to include houses with a common design and most importantly availability of surrounding vegetation. Each sampling season targeted a total of 50 houses per city; a total of 450 houses (50 per season and 150 per city) were sampled in all three cities. Sampling was done in the long-rains, short-rains and dry season for 5 consecutive days. Collection was done between 11:00 am to 3:00 pm daily by a team of three people (one indoor and 2 outdoor) lasting 20 min per house.

Trapped mosquitoes were taken to a temporary field site laboratory in each city and immobilized using triethylamine (TAE), placed in cryovials and immediately preserved in liquid nitrogen for transportation to the laboratory at the International Centre of Insect Physiology and Ecology, Nairobi for identification. Morphological 
identification was done using available taxonomic keys [21-23]. Data on the collection date, species, season and city of collection was captured in Excel. Some mosquitoes that could not be identified to species level, because some of the morphological features were damaged or lost, were classified to genus level as Aedes spp., Mansonia spp. and Culex spp.

\section{Data analysis}

Mosquitoes collected during each of the 5-day trappings per season (i.e. BG-Sentinel collections) from different sites within each city were pooled and counted. We analyzed the total mosquito abundance and specific-species abundance (Ae. aegypti and Ae. bromeliae) using general linear models (GLMs) with seasons and cities as predictors. As a measure of mosquito community structure, we estimated the Shannon diversity index (hereafter referred to as diversity) for each city per trapping season using the vegan package in $\mathrm{R}$ version 3.2.3 [24]. We explored seasonal and city influence on mosquito diversity by applying GLMs after log-transformation to normalize the data. Best-fit models (normal or poisson or quasipoisson or negative binomial generalized linear models) were selected based on model residuals for species richness, diversity, total abundance and species-specific abundance. Data normality was confirmed by performing Shapiro-Wilk tests on model residuals of mosquito diversity. Kilifi was taken as the reference city, and the dry season as the reference season.

For resting mosquito collections, we limited our comparisons to Ae aegypti only. Resting Ae aegypti collected for the different seasons in each city were pooled, and broadly classified as indoors (sitting room, bedroom and kitchen) and outdoors (walls around the house and vegetation). The mosquito resting density estimated as the total number of resting Ae. aegypti by number of collectors was compared, indoors versus outdoors, for each city using the Chi-square test. The proportion of houses positive indoors or outdoors was also compared using the Chi-square test. All data were analyzed in $\mathrm{R}$ version 3.2.3 [24] at $\alpha=0.05$ level of significance.

\section{Results}

\section{Mosquito abundance and composition}

A total of 12,937 mosquitoes representing 6 genera and 25 species were captured throughout the survey from the three cities using the BG-Sentinel traps (Table 1). Aedes aegypti was the most dominant DENV/YFV vector represented across all the cities and seasons except for Nairobi where Aedes tricholabis dominated collections during the long-rains. Kilifi had a wider Aedes species representation (9 spp.) and Mansonia was primarily encountered in Kisumu, especially in the long-rains and dry season. Collections of Culex species were generally low; dominated by Culex pipiens and Culex univittatus in Kisumu during the long-rains, and wider species representation (10 species) in Nairobi. Culex rubinotus was limited to Kilifi, Culex poicilipes to Kisumu and Culex zombaensis to Nairobi, although in low numbers. Toxorhynchites brevipalpis was also recorded in Kilifi and Kisumu, Eretmapodites chrysogaster in Kilifi and Nairobi, while Anopheles species were recorded in all three areas during the long-rains, although in low numbers.

Total mosquito abundance was significantly higher in Kisumu than Kilifi (Estimate $=0.593 \pm 0.29, t=2.08, P=$ 0.043). However, total mosquito abundance did not differ between Kisumu and Nairobi (Estimate $=0.30 \pm 0.27$, $t=1.12, P=0.27$ ) or Kilifi and Nairobi (Estimate $=0.293$ $\pm 0.30, t=0.97, P=0.34$ ) (Table 2). Overall abundance during the long-rains was significantly higher than during the short-rains (Estimate $=2.316 \pm 0.38, t=5.459, P$ $<0.0001$ ) and dry season (Estimate $=2.119 \pm 0.39, t=$ 5.46, $P<0.0001)$, but collections between the short-rains and dry season did not differ significantly (Estimate $=-0.198 \pm 0.51, t=0.39, P=0.7$ ) (Table 2).

Aedes aegypti accounted for 60\% $(n=7772)$ of the total host-seeking mosquitoes collected, with Kilifi yielding 37.7\% ( $n=2932)$, Kisumu 45.2\% $(n=3513)$, and Nairobi $17.1 \%(n=1327)$ (Table 1$)$. While Ae. aegypti abundance in Kilifi and Kisumu were comparable (Estimate $=0.321$ $\pm 0.241, t=1.33, P=0.19$ ), when each was compared to Nairobi, a two-fold and three-fold increase in Ae. aegypti abundance was observed in Kilifi (Estimate $=-0.653 \pm$ 0.32, $t=-2.06, P=0.045$ ), and Kisumu (Estimate $=0.97 \pm$ $0.31, t=3.17, P=0.027$ ), respectively (Table 2). While $A$ e. aegypti abundance varied significantly between the long- and short-rains (Estimate $=2.004 \pm 0.31, t=6.50, P$ $<0.0001$ ), and the long-rains and dry season (Estimate $=$ $2.109 \pm 0.378, t=5.59, P<0.0001)$, the numbers recorded in the short-rains and dry season were not significantly different $\quad($ Estimate $=0.104 \pm 0.46, \quad t=0.23, \quad P=0.82$ ) (Table 2).

Aedes bromeliae was the second most dominant Stegomyia species recorded in all three cities, comprising $0.23 \%(n=29)$ of the total mosquitoes collected, of which $37.9 \%(n=11)$ occurred in Kilifi, $17.2 \%(n=5)$ in Kisumu, and $44.8 \%(n=13)$ in Nairobi. Aedes bromeliae abundance, however, did not vary by city or season (Table 2). Other potential vectors of YFV recorded include Aedes metallicus, Aedes tarsalis and Erytmapodites chrysogaster, although in very low numbers, each representing less than $0.1 \%$ of the total mosquitoes collected (Table 1). Aedes metallicus, Ae. tarsalis and Er. chrysogaster were all recorded in Kilifi, with no record of Er. chrysogaster and Ae. metallicus in Kisumu and Nairobi, respectively. The mosquito species composition encountered throughout the sampling periods in the different cities is shown in Table 1. 
Table 1 Seasonal adult mosquito abundance in Kilifi, Kisumu, and Nairobi between October 2014 and June 2016 using $\mathrm{CO}_{2}$-baited BG-Sentinel traps

\begin{tabular}{|c|c|c|c|c|c|c|c|c|c|}
\hline \multirow[b]{2}{*}{ Mosquito species } & \multicolumn{3}{|l|}{ Kilifi } & \multicolumn{3}{|l|}{ Kisumu } & \multicolumn{3}{|l|}{ Nairobi } \\
\hline & Long-rains & Short-rains & Dry season & Long-rains & Short-rains & Dry season & Long-rains & Short-rains & Dry season \\
\hline Aedes aegyptia & 2235 & 581 & 113 & 2577 & 414 & 522 & 1071 & 180 & 76 \\
\hline Aedes bromeliae ${ }^{b}$ & 6 & 5 & 0 & 0 & 5 & 0 & 13 & 0 & 0 \\
\hline Aedes metallicus ${ }^{\mathrm{b}}$ & 1 & 3 & 0 & 10 & 0 & 0 & 0 & 0 & 0 \\
\hline Aedes tarsalis ${ }^{\mathrm{b}}$ & 3 & 0 & 0 & 0 & 0 & 0 & 0 & 0 & 0 \\
\hline Aedes dentatus & 0 & 1 & 0 & 0 & 0 & 0 & 0 & 0 & 0 \\
\hline Aedes mcintoshi & 2 & 0 & 0 & 50 & 1 & 16 & 101 & 1 & 1 \\
\hline Aedes tricholabis & 57 & 19 & 0 & 1 & 3 & 0 & 2295 & 18 & 6 \\
\hline Aedes hirsutus & 23 & 3 & 0 & 0 & 1 & 0 & 0 & 0 & 0 \\
\hline Aedes longipalpis & 33 & 0 & 0 & 0 & 0 & 0 & 0 & 0 & 0 \\
\hline Aedes spp. & 109 & 1 & 0 & 19 & 0 & 0 & 0 & 39 & 6 \\
\hline Eretmapodites chrysogaster ${ }^{b}$ & 5 & 6 & 0 & 0 & 0 & 0 & 2 & 0 & 0 \\
\hline Mansonia africana & 2 & 0 & 0 & 789 & 39 & 185 & 0 & 0 & 0 \\
\hline Mansonia uniformis & 0 & 0 & 0 & 224 & 7 & 220 & 0 & 0 & 0 \\
\hline Mansonia spp. & 0 & 0 & 0 & 37 & 0 & 0 & 0 & 0 & 0 \\
\hline Culex pipiens & 55 & 4 & 2 & 126 & 1 & 4 & 44 & 5 & 48 \\
\hline Culex annuloris & 0 & 2 & 0 & 3 & 0 & 0 & 33 & 2 & 14 \\
\hline Culex univittatus & 2 & 1 & 0 & 140 & 4 & 0 & 12 & 0 & 3 \\
\hline Cx vansomereni & 3 & 0 & 0 & 0 & 0 & 0 & 21 & 0 & 0 \\
\hline Culex rubinotus & 0 & 1 & 7 & 0 & 0 & 0 & 0 & 0 & 0 \\
\hline Culex zombaensis & 0 & 0 & 0 & 0 & 0 & 0 & 54 & 0 & 10 \\
\hline Culex tigripes & 0 & 0 & 0 & 1 & 0 & 2 & 3 & 0 & 0 \\
\hline Culex poicilipes & 0 & 0 & 0 & 10 & 0 & 0 & 0 & 0 & 0 \\
\hline Culex ethiopicus & 0 & 0 & 0 & 7 & 1 & 0 & 0 & 0 & 3 \\
\hline Culex bitaeniorhynchus & 0 & 0 & 0 & 2 & 0 & 0 & 0 & 3 & 0 \\
\hline Culex spp. & 7 & 2 & 0 & 23 & 0 & 0 & 0 & 2 & 2 \\
\hline Toxorhynchites brevipalpis & 3 & 0 & 0 & 0 & 1 & 0 & 0 & 0 & 0 \\
\hline Anopheles gambiae (s.l.) & 2 & 0 & 0 & 1 & 0 & 0 & 2 & 0 & 0 \\
\hline Anopheles coustani & 0 & 0 & 0 & 1 & 0 & 0 & 0 & 0 & 0 \\
\hline Total & 2548 & 629 & 122 & 4021 & 477 & 949 & 3651 & 250 & 169 \\
\hline
\end{tabular}

a Major vector of DENV and urban YFV

${ }^{\mathrm{b} P o t e n t i a l}$ YFV vectors

\section{Mosquito species richness/diversity}

Of the total 25 species observed in all three areas, 10 belonged to Culex and nine to Aedes (Table 1). Mosquito species richness varied by city and season, being comparable in Kisumu and Nairobi (Estimate $=0.133 \pm$ 1.03, $t=0.13, P=0.90$ ), but significantly higher when each was compared to Kilifi (Nairobi-Kilifi Estimate $=$ $2.168 \pm 0.96, t=2.26, P=0.03$, and Kisumu-Kilifi Estimate $=2.301 \pm 0.96, t=2.40, P=0.02$ ). Mosquito species richness was significantly higher in the long-rains compared to the short-rains (Estimate $=5.77 \pm 0.96, t=6.02$, $P<0.0001$ ) and dry season (Estimate $=6.87 \pm 1.03, t=$ 6.68, $P<0.0001$ ), but not between the short-rains and dry season (Estimate $=1.098 \pm 0.96, t=1.15, P=0.26$ ). Species richness varied from 6 to 20 in Kilifi, 6 to 22 in Kisumu, and 10 to 18 species in Nairobi from the dry to rainy seasons. Also, the overall mosquito diversity varied by city and season. Mean mosquito diversity was twofold higher in Nairobi $(H=1.03)$ compared to Kisumu $(H=0.60$, Estimate $=-0.123 \pm 0.037, t=-3.30, P=0.002)$, and three-fold higher in Nairobi compared to Kilifi ( $H=$ 0.31 , Estimate $=0.186 \pm 0.035, t=5.36, P<0.0001$ ) (Table 2, Fig. 2). Mosquito diversity was, however, not significantly different between Kilifi and Kisumu (Estimate = $0.063 \pm 0.035, t=1.821, P=0.075$ ) (Table 2). We found significantly higher mosquito diversity in the long- 


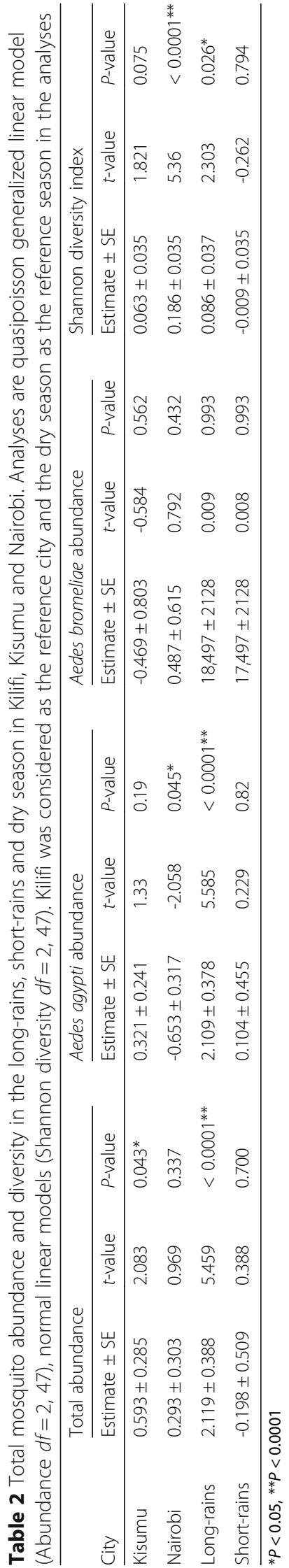




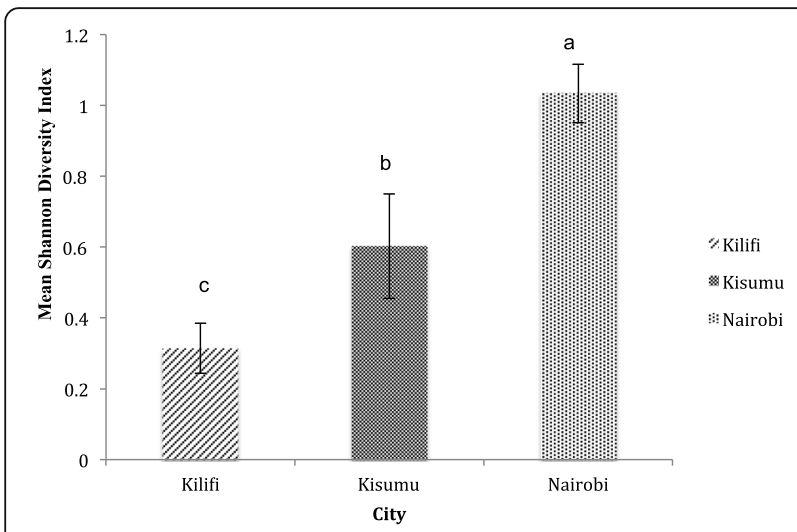

Fig. 2 Mean Shannon diversity index for mosquitoes collected using BG-Sentinel traps in Kilifi, Kisumu and Nairobi in Kenya. Means followed by different letters are significantly different at $a=0.05$

versus short-rains (Estimate $=0.095 \pm 0.035, t=2.73, P=$ 0.009 ), than between the long-rains and dry season (Estimate $=0.086 \pm 0.037, t=2.303, P=0.026$ ), but no difference between the short-rains and dry season (Estimate $=$ $0.009 \pm 0.035, t=0.26, P=0.79$ ) (Table 2). Mosquito diversity ranged from 0.04 to 1.9 , with the lowest value recorded in Kisumu during the dry season and the highest in Nairobi in the dry season.

\section{Resting preferences}

Of the 450 houses (150 per city, 50 per season) inspected from all three cities, $10 \%(n=44)$ were positive for either male or female Ae aegypti. Of these positive houses, $27.3 \%(n=12)$ were from Kilifi, $52.3 \%(n=23)$ from Kisumu and $20.4 \%(n=9)$ from Nairobi. A total of 73 Ae. aegypti only were aspirated from all three cities, $44 \%(n=32)$ females and 56\% $(n=41)$ males both indoors and outdoors. This translated to a resting density of $0-5$ mosquitoes indoors and 6-21 mosquitoes outdoors for the different cities (Table 3). Apart from one
Ae. bromeliae, which was collected outdoors in Kilifi, Ae. aegypti was the only other Stegomyia species sampled in the resting collection. While there was no significant difference in the number of Ae. aegypti found resting indoors and outdoors in Kilifi $\left(\chi^{2}<0.0001, d f=1\right.$, $P=1.0)$, significantly higher numbers were found resting outdoors compared to indoors in Kisumu $\left(\chi^{2}=28.17, d f\right.$ $=1, P<0.0001)$. In Nairobi, resting was exclusively outdoors. Also, in Kilifi, the catches of females $\left(\chi^{2}=0, d f=\right.$ $1, P=1.0)$ either indoors or outdoors, like that of males $\left(\chi^{2}=1, d f=1, P=0.32\right)$, did not differ significantly. However, in Kisumu significantly more females $\left(\chi^{2}=6.25\right.$, $d f$ $=1, P=0.012)$ and males $\left(\chi^{2}=19.2, d f=1, P<0.0001\right)$ were captured outdoors than indoors. Overall, in Kilifi the total number of females aspirated was significantly higher than the number of males $\left(\chi^{2}=6.55, d f=1, P=\right.$ $0.011)$, while in Kisumu $\left(\chi^{2}=3.13, d f=1, P=0.077\right)$ and Nairobi $\left(\chi^{2}=0, d f=1, P=1\right)$, there was no significant difference.

\section{Discussion}

Aedes aegypti, the known DENV vector in Kenya [9], was generally found to be the most abundant mosquito species. Abundance was highest during the long-rains, relative to the short-rains and dry season, and comparably higher in Kilifi and Kisumu vs Nairobi. We found very low occurrence of Ae. bromeliae, a species which did not vary by city or season. Furthermore, variation in mosquito diversity was evident, being highest in Nairobi and during the long-rains (Table 2). Variation in abundance and diversity, both important epidemiological risk parameters, may impact differentially on transmission risk of DENV/YFV across seasons and cities. More outdoor resting was observed for Ae. aegypti, suggesting the existence of an exophilic population especially in $\mathrm{Ki}$ sumu and Nairobi. In Kilifi, resting data are suggestive of a more endophilic population of Ae. aegypti.

Table 3 Indoor and outdoor resting densities of Aedes aegypti mosquitoes collected in Kilifi, Kisumu and Nairobi using Prokopack aspirators from October 2014-June 2016

\begin{tabular}{|c|c|c|c|c|c|c|c|c|}
\hline \multirow[b]{2}{*}{ Area } & \multirow[b]{2}{*}{ Location } & \multirow[b]{2}{*}{ Positive houses $^{\mathrm{b}}$} & \multicolumn{2}{|l|}{ Female } & \multicolumn{2}{|l|}{ Male } & \multicolumn{2}{|l|}{ Total $^{a}$} \\
\hline & & & No. collected & Resting density $^{c}$ & No. collected & Resting density $^{c}$ & $\overline{\text { No. collected }}$ & Resting density ${ }^{c}$ \\
\hline \multirow[t]{2}{*}{ Kilifi } & Indoor $^{d}$ & $3.3\left(0.01-0.08^{*}\right)(n=5)$ & 5 & 5 & 0 & 0 & 5 & 5 \\
\hline & Outdoor $^{\mathrm{e}}$ & $5.3\left(0.03-0.11^{*}\right)(n=8)$ & 7 & 4 & 4 & 2 & 11 & 6 \\
\hline \multirow[t]{2}{*}{ Kisumu } & Indoor & $1.3\left(0.002-0.05^{*}\right)(n=2)$ & 1 & 1 & 1 & 1 & 2 & 2 \\
\hline & Outdoor & $14.0\left(0.09-0.21^{*}\right)(n=21)$ & 14 & 7 & 28 & 14 & 42 & 21 \\
\hline \multirow[t]{2}{*}{ Nairobi } & Indoor & $0\left(0.0-0.03^{*}\right)(n=0)$ & 0 & 0 & 0 & 0 & 0 & 0 \\
\hline & Outdoor & $6.0\left(0.03-0.11^{*}\right)(n=9)$ & 5 & 3 & 8 & 4 & 13 & 7 \\
\hline
\end{tabular}

${ }^{\mathrm{a}}$ Total males and females Ae. aegypti collected

$\mathrm{b}_{\%}$ Positive houses ( $95 \%$ confidence interval) ( $n$, number of positive houses)

${ }^{c}$ Resting density $=$ No. collected/No. of collectors

${ }^{d}$ Indoors: sitting room, bedroom and kitchen

eOutdoor: nearby vegetation and outside walls

${ }^{*} P<0.0001$ 
The Ae. aegypti abundance pattern was strongly correlated with seasonal rainfall, with higher abundance during the long-rains compared to the other periods, in all three sampling cities (Table 1). In fact, 5 and 8 times more $A e$ aegypti were captured during the long-rains compared to short-rains and dry season, respectively (Table 1). This is expected, as the abundance of mosquitoes, including Ae. aegypti, is generally associated with rainfall [25]. Previous findings have found rainfall to be an important driver of Ae. aegypti populations and dengue incidence [25], corroborating the occurrence of dengue outbreaks in Kenya and East Africa during periods of heavy rainfall $[9,26]$. Taken together, our findings suggest higher risk of DENV transmission in Kilifi/Kisumu than Nairobi, particularly during the long-rains. Nonetheless, the persistence of Ae. aegypti throughout the short-rains and dry season suggests that disease transmission could potentially persist throughout the year due to continued presence of the vector, albeit at lower levels.

Factors relating to availability of breeding sites, temperature or altitudinal differences may have influenced the abundance patterns of Ae. aegypti across the cities $[25,27]$. Being a typical container breeder, we previously found an increase in the number of breeding sites in Kilifi and Kisumu in the long-rains, compared to Nairobi [17], which is located at a higher altitude (1661 masl), and has lower average monthly temperatures (22$28{ }^{\circ} \mathrm{C}$ ), compared to Kilifi (50 masl, $27-31{ }^{\circ} \mathrm{C}$ ) and $\mathrm{Ki}$ sumu (1131 masl, $28-30{ }^{\circ} \mathrm{C}$ ). This may partly explain the low Ae. aegypti abundance found in Nairobi, as significant reductions in Ae. aegypti abundance with an increase in altitude have previously been reported [27]. The same study identified temperature to be a positive risk factor for Ae. aegypti abundance, with vector abundance increasing with an increase in temperature [27]. Autochthonous cases of dengue can be facilitated by local Aedes vectors. However, despite Nairobi being a major hub in East Africa, there no outbreak of dengue has been reported. A possible contributing factor to this pattern could be low Aedes abundance, as was observed in our study, among other factors. The high vector abundance in Kilifi and Kisumu corroborates with their increased breeding sites, especially during the long-rains. This high Ae. aegypti abundance in Kilifi may explain the dengue epidemics reported in this region [7-10]. Also, the high abundance in Kisumu may explain the recent reports of sporadic cases of dengue (R. Sang, unpublished data), although outbreaks have not been reported. The high abundance of Ae aegypti in Kisumu, and its potential role in dengue epidemics, is therefore deserving of further consideration.

Aedes bromeliae, Ae. metallicus, Ae. tarsalis and Er. chrysogaster are sylvatic vectors mostly found inhabiting discarded plastic containers as well as natural containers (tree holes, rock pools and discarded coconut shells) [17]. These YFV vectors have been implicated in yellow fever outbreaks in East and Central Africa [28-31]. Trap captures for these species were generally low and it is not certain if this could be related to potential sampling bias of the BG-Sentinel trap employed for sampling. This trap, whilst designed to target Ae. aegypti [32, 33], has been shown to effectively collect other disease vectors including Anopheles species and even sandflies [34, 35]. As such, it appears unlikely that this trapping tool may have affected the overall mosquito diversity and abundance, particularly as we also baited the traps with $\mathrm{CO}_{2}$, which is thus far known to be the most potent attractant to mosquito species [36, 37]. However, given that in our previous study [17] we encountered high numbers of Ae. bromeliae and Er. chrysogaster immatures, the low numbers of adults recorded in this study suggest that adults may be poorly attracted to the BG-Sentinel trap. This indicates that developing better sampling tools for targeting adults of these species is an important consideration. In addition, the introduction of sylvatic YFV vectors into urban areas, as was observed in this study, is of public health concern. The YFV could adapt to these vectors given their ability to act as potential enzootic vectors. Their ability to transmit the YFV therefore warrants further assessment.

Although species richness was comparable in Nairobi and Kisumu, Nairobi had the highest overall diversity of mosquitoes (Fig. 2). The species observed in Kilifi, especially Ae. bromeliae, Ae. metallicus, Ae. tarsalis and Er. chrysogaster, which in addition to Ae. aegypti, are known vectors for YFV, should not be ignored. These species may be of epidemiological value, in the light of pathogen adaptation to multiple vectors as observed for chikungunya virus in Senegal $[38,39]$. Their role in sustaining an outbreak of dengue and chikungunya therefore needs to be assessed, as these vectors, especially Ae. bromeliae, could serve as bridge vectors [40], moving the virus from the sylvatic to the urban transmission cycle. While mosquito diversity was higher during the rainy seasons compared to the dry season in Kilifi and Kisumu, the observed pattern was different in Nairobi, where higher mosquito diversity was recorded in the dry season. The diversity pattern in Nairobi may have been influenced by the Culex collections, which were fairly represented in Nairobi with only sparse occurrence in Kilifi/Kisumu, especially during the dry season. Although Culex species are not important vectors in the epidemiology of DENV/ YFV, they play an important role in the transmission of other arboviruses, such as West Nile virus [41].

Despite extensive sampling effort, we found very low numbers of resting Ae. aegypti mosquitoes, both indoors and outdoors. The sampling regime and effort is in line 
with techniques employed elsewhere [42, 43]. The low numbers of Ae aegypti resting indoors is in stark contrast to the largely indoor habit known for this species in Asia and South America [42, 44, 45]. This suggests a difference in the resting patterns of Ae. aegypti found in Kenya and corroborates findings from previous studies reporting generally low numbers of resting Ae. aegypti in this region $[9,46]$. The largely outdoor resting habit of this species concurs with its breeding pattern being mainly outdoors $[9,17,46]$. However, the low outdoor numbers recorded here suggest other resting sites, apart from vegetation, that require further elucidation. Although most of the outdoor resting was observed on vegetation, this study did not investigate in detail the preferred plant types, as this was not within the scope of the study. Overall, the knowledge of these resting patterns can be exploited in emergency operations targeting adults to break transmission in an outbreak situation. The proportion of adults found resting indoor/outdoor varied between Kilifi and the other cities; for Nairobi and Kisumu it was largely outdoors. This finding indicates possible population differences in resting habits among these cities, which is worth exploring.

\section{Conclusions}

Aedes aegypti was the most dominant mosquito species recorded and its occurrence varied by city and season. The abundance pattern suggests that the risk of DENV transmission is elevated during the long-rains and in Kilifi/Kisumu compared to Nairobi, assuming that the vector populations are similarly anthropophagic and efficient in transmitting the virus, but this is still under investigation. The low abundance of Ae. bromeliae recorded is suggestive of a low risk of YFV transmission in all three cities. The overall mosquito abundance pattern neither correlated with species richness nor diversity. In addition to vector abundance, feeding habits and vector competence are factors that can differentially drive the emergence of dengue and yellow fever in an area. Therefore, to fully understand the risk associated with the transmission of DENV/YFV in these cities of Kenya, these factors need to be assessed. The resting pattern for Ae. aegypti is suggestive of a more endophilic population in Kilifi, and an exophilic population in Kisumu and Nairobi. This knowledge on the resting behavior can be exploited in adult mosquito control to break transmission during emergency outbreak situations. Continuous vector surveillance should, however, be routinely performed for early detection of changing vector dynamics that may precipitate an outbreak of dengue/ yellow fever.

\section{Abbreviations}

DENV: dengue virus; GLM: general linear models; TEA: triethylamine; YFV: yellow fever virus

\section{Acknowledgements}

We thank Joel Lutomiah of the Kenya Medical Research Institute (KEMRI, Nairobi) for study site selection and Caroline Tigoi (ICIPE, Nairobi) for project management. We also thank John Gachoya, Dunstone Beti, Reuben Lugali (KEMRI), Francis Mulwa, James Wauna, Mwaura Kageche (ICIPE) for mosquito collection and identification. We acknowledge Jackson Kimani, GIS support unit, ICIPE for producing the map of the study area. We are also grateful for the support from the local chiefs and members of the Githogoro, Kajulu, Kanyarkwar, Nyalenda B, and Rabai communities.

\section{Funding}

We gratefully acknowledge the financial support for this research by the following organizations and agencies: National Institutes of Health $(\mathrm{NIH})$, Grant No. 1R01Al099736-01A1 to RS; UK Aid from the UK Government; Swedish International Development Cooperation Agency (Sida); the Swiss Agency for Development and Cooperation (SDC); and the Kenyan Government. SBA was supported by a German Academic Exchange Service (DAAD) In-Region Postgraduate Scholarship. The views expressed herein do not necessarily reflect the official opinion of the donors.

Availability of data and materials

All data generated or analyzed during this study are included in this published article.

\section{Authors' contributions}

SBA, DPT, ADSB and RS conceived and designed experiments. SBA conducted the experimental work. SBA and DPT analysed the data. SBA drafted the manuscript with subsequent contributions and revisions from DPT, ADSB and RS. All authors read and approved the final manuscript.

Ethics approval and consent to participate

We sought permission from household heads through oral informed consent to allow their residences to be surveyed for resting mosquitoes. Household survey of mosquitoes was carried out with ethical approval from Kenya Medical Research Institute Scientific and Ethics Review Unit (KEMRISERU) (Project Number SERU 2787).

Consent for publication

Not applicable.

Competing interests

The authors declare that they have no competing interests.

\section{Publisher's Note}

Springer Nature remains neutral with regard to jurisdictional claims in published maps and institutional affiliations.

\section{Author details}

${ }^{1}$ International Centre of Insect Physiology and Ecology, P. O Box 30772-00100, Nairobi, Kenya. ' ${ }^{2}$ Department of Zoology and Entomology, University of Pretoria, Private Bag 20, Hatfield 0083, South Africa. ${ }^{3}$ Arbovirus/ Viral Hemorrhagic Fever Laboratory, Centre for Virus Research, Kenya Medical Research Institute, P. O Box 54840-00200, Nairobi, Kenya.

Received: 29 August 2017 Accepted: 17 December 2017

Published online: 29 December 2017

\section{References}

1. Gubler DJ. Dengue and dengue hemorrhagic fever. Clin Microbiol Rev. 1998;11(3):480-96

2. Kraemer MUG, Faria NR, Reiner RC, Golding N, Nikolay B, Stasse S, et al. Spread of yellow fever virus outbreak in Angola and the Democratic Republic of the Congo 2015-16: a modelling study. Lancet Infect Dis. 2017; 17(3):330-8.

3. Sang RC. Dengue in Africa. In: report of the scientific working group meeting on dengue. Geneva: WHO special programme for research and training in tropical. diseases. 2007:50-2. http://apps.who.int/iris/bitstream/ 10665/69787/1/TDR_SWG_08_eng.pdf

4. Wilder-Smith A, Monath TP. Responding to the threat of urban yellow fever outbreaks. Lancet Infect Dis. 2016;17:248-50. 
5. Bhatt $\mathrm{S}$, Gething PW, Brady OJ, Messina JP, Farlow AW, Moyes CL, et al. The global distribution and burden of dengue. Nature. 2013;496(7446):504-7.

6. Katzelnick LC, Fonville JM, Gromowski GD, Arriaga JB, Green A, James SL, et al. Dengue viruses cluster antigenically but not as discrete serotypes. Science. 2015;349(6254):1338-43.

7. Ellis EM, Neatherlin JC, Delorey M, Ochieng M, Mohamed AH, Mogeni DO, et al. A household serosurvey to estimate the magnitude of a dengue outbreak in Mombasa, Kenya, 2013. PLoS Negl Trop Dis. 2015;9(4):e0003733.

8. Johnson BK, Ocheng D, Gichogo A, Okiro M, Libondo D, Kinyanjui P, et al. Epidemic dengue fever caused by dengue type 2 virus in Kenya: preliminary results of human virological and serological studies. East Afr Med J. 1982; 59(12):781-4.

9. Lutomiah J, Barrera R, Makio A, Mutisya J, Koka H, Owaka S, et al. Dengue outbreak in Mombasa city, Kenya, 2013-2014: entomologic investigations. PLoS Negl Trop Dis. 2016;10(10):e0004981.

10. Konongoi L, Ofula V, Nyunja A, Owaka S, Koka H, Makio A, et al. Detection of dengue virus serotypes 1, 2 and 3 in selected regions of Kenya: 20112014. Virol J. 2016;13:182

11. Wasserman S, Tambyah PA, Lim PL. Yellow fever cases in Asia: primed for an epidemic. Int J Infect Dis. 2016;48:98-103.

12. World Health Organisation. Emergencies: Yellow fever situation report. 2017. http://www.who.int/emergencies/yellow-fever/situation-reports/2-june-2016/en/.

13. Reiter P, Cordellier R, Ouma JO, Cropp CB, Savage HM, Sanders EJ, et al. First recorded outbreak of yellow fever in Kenya, 1992-1993. II. Entomologic investigations. Am J Trop Med Hyg. 1998:59(4):650-6.

14. World Health Organisation. Yellow fever situation report. WHO; 2016. http:// www.who.int/emergencies/yellow-fever/situation-reports/30-june-2016/en/.

15. Lutomiah J, Bast J, Clark J, Richardson J, Yalwala S, Oullo D, et al. Abundance, diversity, and distribution of mosquito vectors in selected ecological regions of Kenya: public health implications. J Vector Ecol. 2013;38(1):134-42.

16. World Health Organisation. Immunization, vaccines and biological: Questions and answers on dengue vaccines. WHO. 2017. http://www.who. int/immunization/research/development/dengue_q_and_a/en/.

17. Agha SB, Tchouassi DP, Bastos ADS, Sang R. Assessment of risk of dengue and yellow fever virus transmission in three major Kenyan cities based on Stegomyia indices. PLoS Negl Trop Dis. 2017;11(8):e0005858.

18. Anders $\mathrm{KL}$, Hay SI. Lessons from malaria control to help meet the rising challenge of dengue. Lancet Infect Dis. 2012;12(12):977-84.

19. Brown JE, McBride CS, Johnson P, Ritchie S, Paupy C, Bossin H, et al. Worldwide patterns of genetic differentiation imply multiple "domestications" of Aedes aegypti, a major vector of human diseases. Proc R Soc B Biol Sci. 2011;278(1717):2446-54.

20. Carrington LB, Simmons CP. Human to mosquito transmission of dengue viruses. Front Immunol. 2014:5:290.

21. Edwards FW. Mosquitoes of the Ethiopian Region III. - Culicine adults and pupae. London: British Museum (Natural History); 1941.

22. Gillies MT, Coetzee MA. Supplement to the Anophelinae of Africa south of the Sahara. Afrotropical Region. 1987;55:1-143.

23. Jupp PG. Mosquitoes of southern Africa. South Africa: Ekogilde Publishers; 1996.

24. The R Core Team version 3.2.3. R: A language and environment for statistical computing. Vienna: R foundation for statistical computing; 2015.

25. Barrera R, Amador M, Mackay AJ. Population dynamics of Aedes aegypti and dengue as influenced by weather and human behavior in San Juan, Puerto Rico. PLoS Negl Trop Dis. 2011;5(12):e1378.

26. Mboera LEG, Mweya CN, Rumisha SF, Tungu PK, Stanley G, Makange MR, et al. The risk of dengue virus transmission in Dar es Salaam, Tanzania during an epidemic period of 2014. PLoS Negl Trop Dis. 2016;10(1):e0004313.

27. Dhimal M, Gautam I, Joshi HD, O'Hara RB, Ahrens B, Kuch U. Risk factors for the presence of chikungunya and dengue vectors (Aedes aegypti and Aedes albopictus), their altitudinal distribution and climatic determinants of their abundance in central Nepal. PLoS Negl Trop Dis. 2015:9:e0003545.

28. Ellis BR, Barrett ADT. The enigma of yellow fever in East Africa. Rev Med Virol. 2008;18(5):331-46.

29. Fontenille $D$, Diallo $M$, Mondo $M$, Ndiaye $M$, Thonnon J. First evidence of natural vertical transmission of yellow fever virus in Aedes aegypti, its epidemic vector. Trans R Soc Trop Med Hyg. 1997;91(5):533-5.

30. World Health Organistion. Yellow fever: rapid field entomological assessment during yellow fever outbreaks in Africa: handbook: methodological field approaches for scientists with a basic background in entomology. 2014. http://www.who.int/iris/handle/10665/112785.
31. Serie C, Andral L, Casals J, Williams MC, Brès P, Neri P. Studies on yellow fever in Ethiopia. 5. Isolation of virus strains from arthropod vectors. Bull World Health Organ. 1968;38(6):873-7.

32. Krockel U, Rose A, Eiras AE, Geier M. New tools for surveillance of adult yellow fever mosquitoes: comparison of trap catches with human landing rates in an urban environment. J Am Mosq Control Assoc. 2006;22(2):22938.

33. Williams CR, Long SA, Webb CE, Bitzhenner M, Geier M, Russell RC, et al. Aedes aegypti population sampling using BG-sentinel traps in North Queensland Australia: statistical considerations for trap deployment and sampling strategy. J Med Entomol. 2007:44(2):345-50.

34. Farajollahi A, Kesavaraju B, Price DC, Williams GM, Healy SP, Gaugler R, et al. Field efficacy of BG-sentinel and industry-standard traps for Aedes albopictus (Diptera: Culicidae) and West Nile virus surveillance. J Med Entomol. 2009; 46(4):919-25.

35. Hoel DF, Kline DL, Hogsette JA, Bernier UR, El-Hossary SS, Hanafi HA, et al. Efficacy of commercial mosquito traps in capturing Phlebotomine sand flies (Diptera: Psychodidae) in Egypt. J Med Entomol. 2010;47(6):1179-84.

36. Gillies MT. The role of carbon dioxide in host-finding by mosquitoes (Diptera: Culicidae): a review. Bull Entomol Res. 1980;70(4):525-32.

37. Tchouassi DP, Okiro ROK, Sang R, Cohnstaedt LW, McVey DS, Torto B. Mosquito host choices on livestock amplifiers of Rift Valley fever virus in Kenya. Parasit Vectors. 2016;9:184

38. Diallo D, Sall AA, Buenemann M, Chen R, Faye O, Diagne $C T$, et al. Landscape ecology of sylvatic chikungunya virus and mosquito vectors in southeastern Senegal. PLoS Negl Trop Dis. 2012;6(6):e1649.

39. Diallo M, Thonnon J, Traore-Lamizana M, Fontenille D. Vectors of chikungunya virus in Senegal: current data and transmission cycles. Am J Trop Med Hyg. 1999;60(2):281-6.

40. Haddow AJ. The natural history of yellow fever in Africa. Proc R Soc Edinb Sect B Biol Sci. 1969;70(3):191-227.

41. Lutomiah JL, Koka H, Mutisya J, Yalwala S, Muthoni M, Makio A, et al. Ability of selected Kenyan mosquito (Diptera: Culicidae) species to transmit West Nile virus under laboratory conditions. J Med Entomol. 2011;48(6):1197-201.

42. Chadee DD. Resting behaviour of Aedes aegypti in Trinidad: with evidence for the re-introduction of indoor residual spraying (IRS) for dengue control. Parasit Vectors. 2013:6:255

43. Vazquez-Prokopec GM, Galvin WA, Kelly R, Kitron UA. New, cost-effective, battery-powered aspirator for adult mosquito collections. J Med Entomol. 2009;46(6):1256-9

44. Dzul-Manzanilla F, Ibarra-López J, Bibiano Marín W, Martini-Jaimes A, Leyva JT, Correa-Morales F, et al. Indoor resting behavior of Aedes aegypti (Diptera: Culicidae) in Acapulco, Mexico. J Med Entomol. 2017;54(2):501-4.

45. Scott TW, Amerasinghe PH, Morrison AC, Lorenz LH, Clark GG, Strickman D, et al. Longitudinal studies of Aedes aegypti (Diptera: Culicidae) in Thailand and Puerto Rico: blood feeding frequency. J Med Entomol. 2000;37(1):89-101.

46. Yalwala S, Clark J, Oullo D, Ngonga D, Abuom D, Wanja E, et al. Comparative efficacy of existing surveillance tools for Aedes aegypti in western Kenya. J Vector Ecol. 2015;40(2):301-7.

\section{Submit your next manuscript to BioMed Central and we will help you at every step:}

- We accept pre-submission inquiries

- Our selector tool helps you to find the most relevant journal

- We provide round the clock customer support

- Convenient online submission

- Thorough peer review

- Inclusion in PubMed and all major indexing services

- Maximum visibility for your research

Submit your manuscript at www.biomedcentral.com/submit 